\title{
Mutation analysis of CACNA1S and SCN4A in patients with hypokalemic periodic paralysis
}

\author{
XIAO-YING WANG ${ }^{1,2}$, BING-WEN REN ${ }^{3}$, ZENG-HUA YONG ${ }^{1}$, \\ HONG-YAN XU ${ }^{1}$, QIU-XIA FU ${ }^{1}$ and HE-BIN YAO ${ }^{1}$ \\ ${ }^{1}$ Department of Endocrinology, Chinese People's Liberation Army Navy General Hospital, Beijing 100048; \\ ${ }^{2}$ Outpatient Clinic of Chinese People's Liberation Army Navy Department, Beijing 100841; \\ ${ }^{3}$ Department of Cadres Medical Care, Chinese People's Liberation Army Navy General Hospital, Beijing 100048, P.R. China
}

Received November 18, 2014; Accepted July 22, 2015

DOI: $10.3892 / \mathrm{mmr} .2015 .4201$

\begin{abstract}
Mutations in CACNA1S (calcium channel, voltage-dependent, L type, alpha $1 \mathrm{~S}$ subunit) and SCN4A (sodium channel, voltage-gated, type IV, alpha subunit) are associated with hypokalemic periodic paralysis (HPP). The aim of the current study was to investigate CACNA1S and SCN4A mutations in patients with HPP. Mutations in CACNA1S and SCN4A were detected in three familial hypokalemic periodic paralysis (FHPP) pedigrees and in two thyrotoxic hypokalemic periodic paralysis (THPP) pedigrees using polymerase chain reaction, DNA sequencing and sequence alignment with GenBank data. A single base mutation from cytosine to guanine at site 1582 was identified in exon 11 of CACNA1S in one FHPP pedigree, resulting in an arginine to glycine (R528G) substitution. A single base mutation from thymine to cytosine at site 2012 was identified in exon 12 of SCN4A in one THPP pedigree, resulting in a phenylalanine to serine (F671S) substitution. No mutations in CACNA1S or SCN4A were identified in the remaining three pedigrees. The present study indicated that CACNA1S and SCN4A mutations are relatively rare in patients with HPP, and further studies are required to determine whether these mutation-associated substitutions are representative of patients with HPP.
\end{abstract}

\section{Introduction}

Hypokalemic periodic paralysis (HPP) is characterized by recurrent attacks of hypokalemia and muscle weakness and is widely recognized as a typical skeletal muscle channelopathy. Primary HPP is categorized into either familial HPP (FHPP) or sporadic HPP (SHPP). Thyrotoxic HPP (THPP) is representative

Correspondence to: Professor He-Bin Yao, Department of Endocrinology, Chinese People's Liberation Army Navy General Hospital, 6 Fucheng Road, Beijing 100048, P.R. China

E-mail: yhb196321@163.com

Key words: hypokalemic periodic paralysis, mutation, CACNA1S, SCN4A of all secondary HPP conditions and although familial cases have been reported, the majority cases of THPP are sporadic.

According to pedigree analysis, FHPP is an autosomal dominant disease with lower penetrance in females (1). The pathogenesis of FHPP is attributed to mutations in genes encoding ion channels in skeletal muscle, although the underlying mechanism remains unclear. Associated mutations have been most commonly reported in the genes encoding the voltage-dependent $\mathrm{L}$ type calcium channel subunit alpha $1 \mathrm{~S}$ (CACNA1S) and the voltage-gated sodium channel type IV alpha subunit (SCN4A) $(2,3)$. Several point mutations $(4,5)$ in CACNA1S and SCN4A have been identified to have associations with FHPP, including Arg1239His/Gly and Arg528His in CACNA1S, and Arg669His, Arg672His/Gly and Arg672Ser in SCN4A. Additional rare substitutions have also been reported (6). Some overlap in the mutations in FHPP, SHPP (7), and THPP (8) has been reported.

To date, the majority of genetic studies of HPP have been conducted in Western populations $(2,6)$. Fewer HPP cases have been reported in China compared with Western countries, and information on HPP in Chinese populations remains limited $(2,6)$. The aim of the current study was to screen for mutations in CACNA1S and SCN4A in Chinese patients with HPP. The present study aimed to determine whether mutations associated with HPP in Chinese cases are the same as those in Western populations, and to provide further epidemiological evidence for HPP.

\section{Materials and methods}

Study subjects. Informed consent was obtained from all participating patients and the study was approved by the medical ethics committee of the Chinese People's Liberation Army Navy General Hospital (Beijing, China). In total, three FHPP and two THPP pedigrees were investigated and all patients involved were free from any other inherited systemic diseases or congenital disorders and presented with typical periodic attacks of limb paralysis with severe hypokalemia. The pedigree analysis is presented in Fig. 1.

Reagents and materials. High fidelity Pyrobest DNA polymerase and dNTPs were purchased from Takara Bio, Inc. 
Table I. Polymerase chain reaction primers designed for analysis of common mutation sites.

\begin{tabular}{llcc}
\hline Gene & \multicolumn{1}{c}{ Primers (5'-3') } & Amplified sites & Fragment length \\
\hline CACNA1S & L:CACTGAGATGCTGATGAAGATGTA & 528 & 192 bp \\
& R:GCACTCACTTGGTGATCTTGAA & & 181 bp \\
L:ATGAGAGTGCCCGCATCTCC & 1239 & 250 bp \\
R:CTGTTGCACCTGGAAGGACTTG & & 669,672 \\
& L:CTCTGTGACAGGGCCTCATG & & \\
\hline
\end{tabular}

L, left primer; R, right primer; bp, base pairs.

Table II. Polymerase chain reaction primers for amplification of CACNA1S and SCN4A exons.

\begin{tabular}{llc}
\hline Exon number & Left primer & Right primer \\
\hline CACNA1S & & GGGAAGTCTGGGCAAGG \\
11 & GGGAGTCAGGAGAATGG & CCTTGCCGCTGCTCACT \\
20 & CCAGGCTGCTGCCTCTT & TCCTTGTGCTTGAGAGT \\
21 & ACAGGCCTGTTCTCCAC & TCCTTGTGCTTGAGAGT \\
22 & ACAGGCCTGTTCTCCAG & AATAAACCATAAGTGCC \\
26 & CTGTGATAACTCAATGG & CCAGGTACGTGCAGTTT \\
30 & GCCCCTTACCCCTCTGT & \\
SCN4A & & GCTGCCTCTCAAACGCC \\
5 & GACCCTGTGGTACCCCT & GAAGACCCGCAGCAGAC \\
12 & CTACGCTCCTTCAGTCT & CCAGAGGCCCCTTCAGC \\
19 & ACGCACTGATCCCCTCG & GTAGAGGTTCACCTCGT \\
\hline
\end{tabular}

(Otsu, Japan), and Wizard ${ }^{\mathrm{TM}}$ Genomic DNA Purification kits, T4 DNA ligase, plasmid pGEM-T and bacteria JM109 were all purchased from Promega Corporation (Madison, WI, USA).

Mutation analysis. Blood samples from confirmed HPP patients were obtained, and genomic DNA was isolated from white blood cells using a Genomic DNA Purification kit according to the manufacturers instructions. Sequence analysis was conducted to identify previously reported common and infrequent mutation sites in CACNA1S and SCN4A. CACNA1S fragments containing the sequences encoding Arg528 and Arg1239 and a SCN4A fragment containing the sequence encoding Arg669 and Arg672 were amplified by polymerase chain reaction (PCR) with the primers presented in Table I. For the pedigrees free from mutations in the above common sites, alternative PCR primers, presented in Table II, were designed to specifically amplify other fragments in CACNA1S and SCN4A. All primers were synthesized by AuGCT Biotechnology Co., Ltd. (Beijing, China). The PCR products were separated by $2 \%$ agarose gel electrophoresis (Beijing Huahai Henghui Technology Co., Ltd.) and collected using a gel extraction kit (Promega Corporation). The PCR products were used as templates for subsequent PCR expansion, following which the products were collected again. PCR was performed using a reaction solution containing $50 \mu \mathrm{l}$ PCR buffer containing $100 \mu \mathrm{mol} / 1 \mathrm{dNTPs}, 0.2 \mu \mathrm{mol} / 1$ primers, 2 units Taq DNA polymerases and $50 \mathrm{ng}$ DNA template. The cycling conditions consisted of one cycle of denaturation at a temperature of $94^{\circ} \mathrm{C}$ for $5 \mathrm{~min}$, followed by denaturation at a temperature of $94^{\circ} \mathrm{C}$ for $30 \mathrm{sec}$, and annealing at a temperature of $55^{\circ} \mathrm{C}$ for $30 \mathrm{sec}$ and extension at a temperature of $72^{\circ} \mathrm{C}$ for $30 \mathrm{sec}$, for a total of 30 cycles. A final extension step was performed at a temperature of $72^{\circ} \mathrm{C}$ for $5 \mathrm{~min}$. All amplified products were sequenced using an ABI PRISM ${ }^{\circledR} 377$ DNA Sequencer (Thermo Fisher Scientific, Inc., Waltham, MA, USA). Sequencing results were BLAST searched (blast.ncbi. nlm.nih.gov/) against sequences in GenBank.

\section{Results}

Clinicalfeatures. The patients selected for the current study all displayed clinical symptoms in accordance with the diagnostic criteria of HPP. All patients were suffering from irregular muscle weakness with hypokalemia and muscle weakness predominantly in the limbs. Potassium supplements had been effective for all the probands of these pedigrees. Patients in pedigree $\mathrm{D}$ and pedigree $\mathrm{E}$ also exhibited symptoms of hyperthyroidism, with the hyperthyroidism confirmed by thyroid 
A

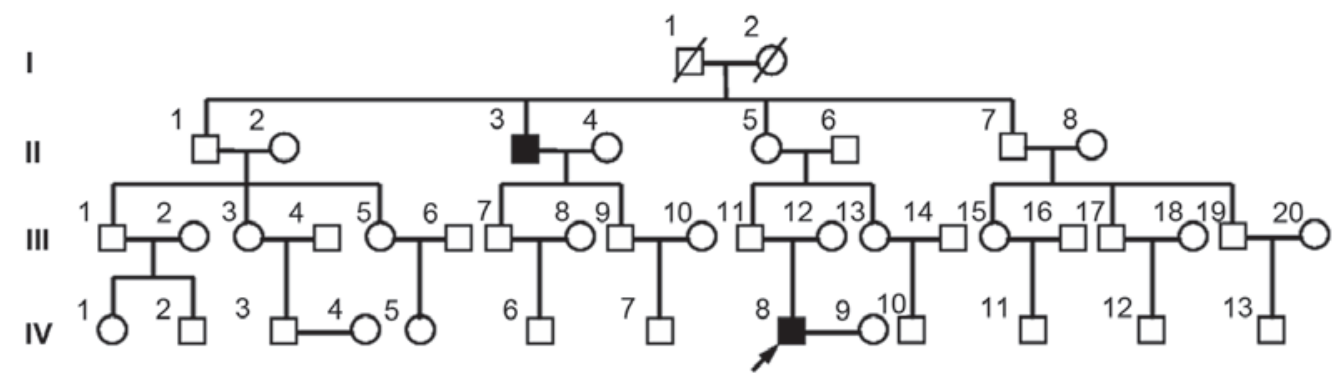

B
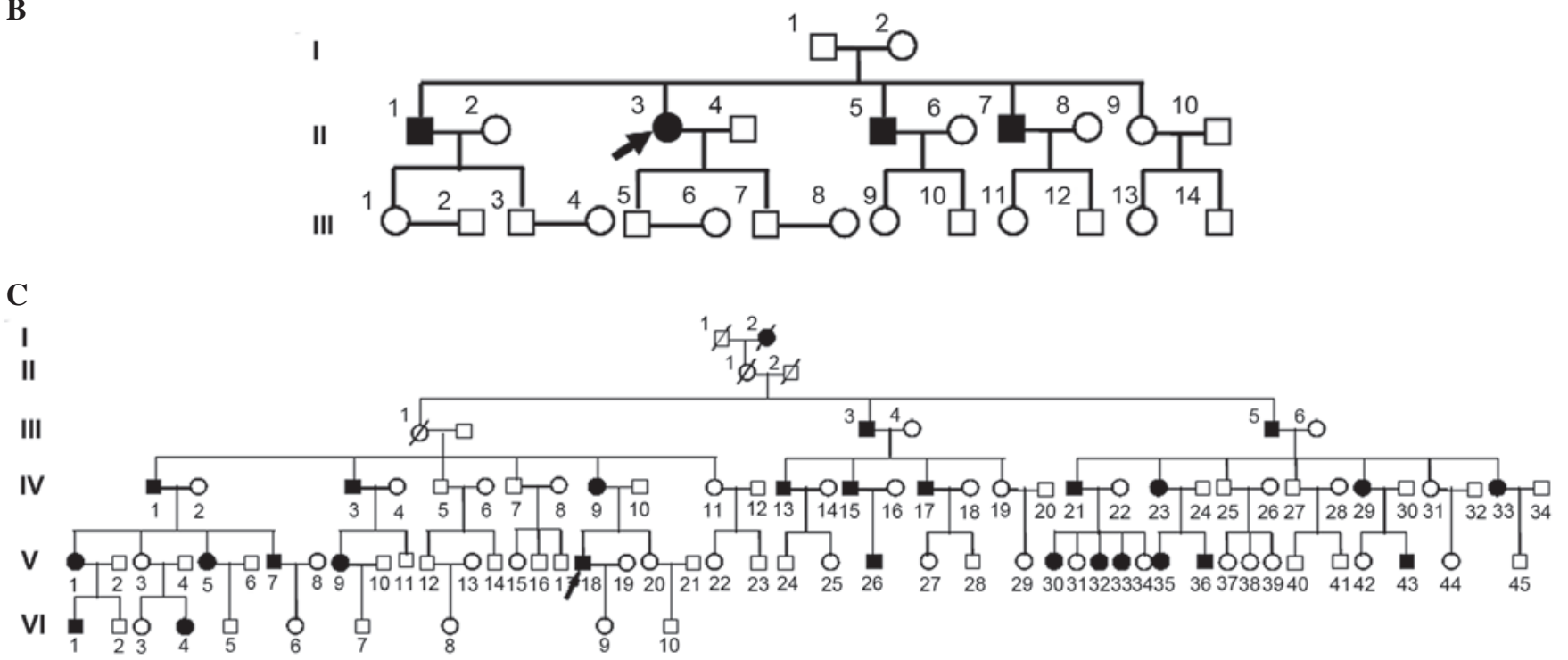

D

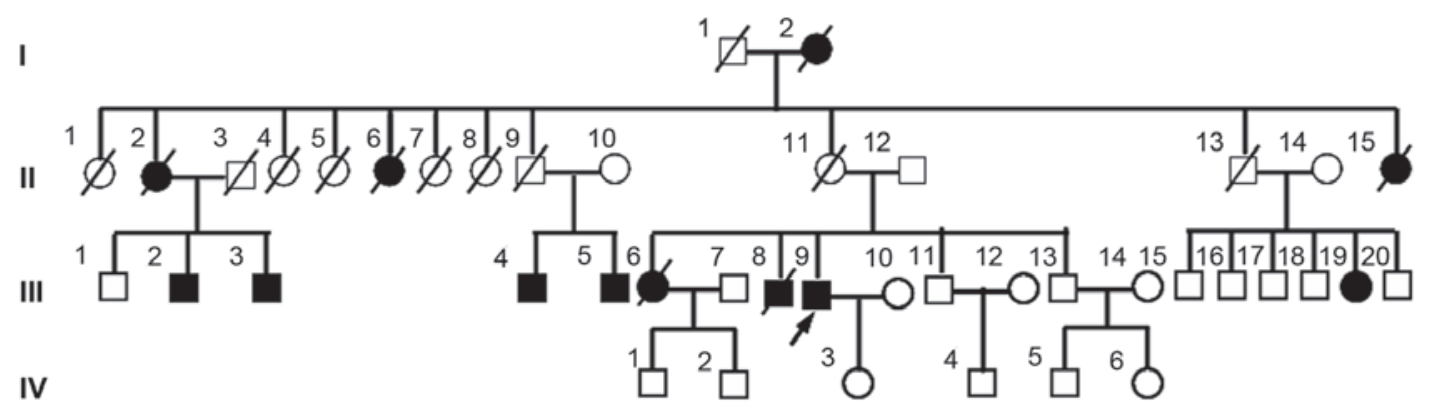

$\mathbf{E}$

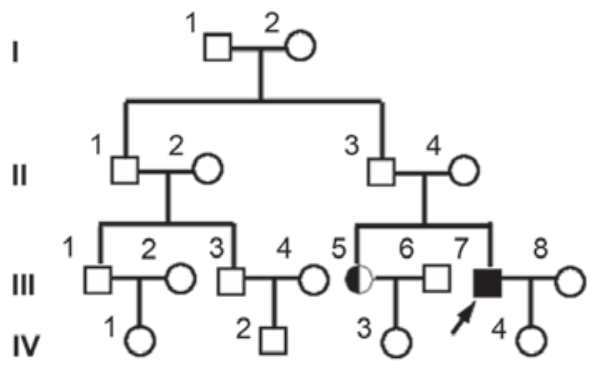

Figure 1. HPP pedigrees. (A) Chinese HPP pedigree A. (B) Chinese HPP pedigree B. (C) Chinese HPP pedigree C. (D) Chinese THPP pedigree D. (E) Chinese THPP pedigree E. Open square, healthy male; open circle, healthy female; filled square, male patient; filled circle, female patient; arrow, proband; crossed out symbol, deceased individual. HPP, hypokalemic periodic paralysis; THPP, thyrotoxic hypokalemic periodic paralysis.

function analysis. The probands were IV8 of pedigree A, II 3 of pedigree $\mathrm{B}, \mathrm{V} 18$ of pedigree $\mathrm{C}$, III9 of pedigree $\mathrm{D}$ and III7 of pedigree $\mathrm{E}$, and their ages of onset were $22,40,15$, 45 and 43 years old, respectively. As presented in Fig. 1, the ratio of male to female patients in these pedigrees was 2:0, 3:1,
14:13, 6:6 and 1:1, respectively, and atavism was observed in all five pedigrees.

Results of DNA sequencing. Mutation analysis was first conducted in probands and healthy controls from each 


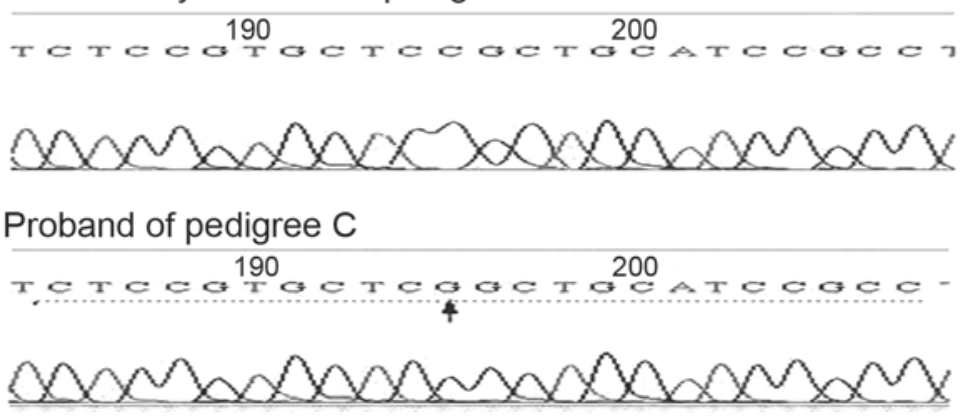

B
Proband:
1 cactgagatgctgatgaagatgtacgggctgggcctgcgccagtact tcatgtctatctt |||||||||||||||||||||||||||||||||||||||||||||||||||||||||||||
Healthy control:
54 cactgagatgctgatgaagatgtacgggctgggcctgcgccagtact tcatgtctatctt 113

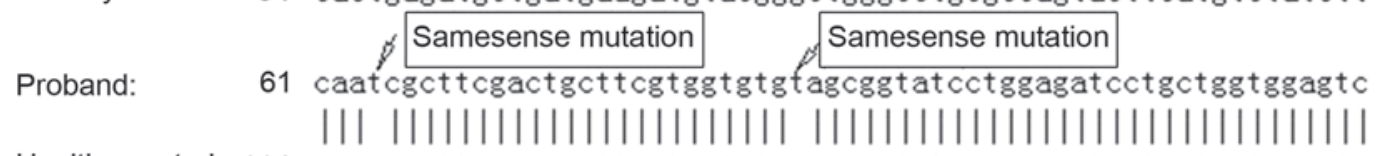
Healthy control: 114 caaccgct cgactgcttcgtggtgtgcagcggtatcctggagatcctgctggtggagtc 173
Proband: 121 gggcgccatgacacccctgggcatctccgtgctcggctgcatccgctcctgaggatctt 180

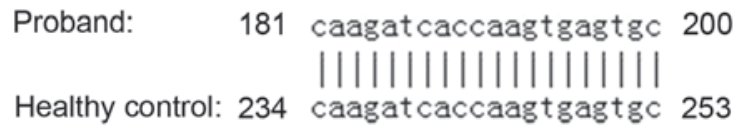

C

Proband: TEMLMKMYGLGLRQ YFMSIFNRFDCFVVCSGILEILLVESG AMTPLGISVLGCIRLLRIFKIT
Healthy control: TEMLMKMYGLGLRQ YFMSIFNRFDCFVVCSGILEILLVESG AMTPLGISVLRCIRLLRIF KIT

Figure 2. Sequence analysis of the CACNA1S region encoding amino acid site 528 in pedigree C. (A) Sequencing maps of the amplified fragment in the proband and one healthy member of pedigree C. (B) Sequence alignment. The sequence of the healthy control from GenBank. (C) The change in amino acid sequence resulting from the missense mutation of cytosine to guanine at nucleotide 1582 in CACNA1S. CACNA1S, voltage-dependent L type calcium channel subunit alpha $1 \mathrm{~S}$.

pedigree. Gene fragments of CACNA1S, containing the 528 and 1239 mutation sites, and of SCN4A, containing the 669 and 672 mutation sites were sequenced. In pedigree $C$ (Fig. 2A), a cytosine to guanine mutation was identified at site 195 in the 192 base pair amplified fragment containing amino acid site 528 of CACNA1S. Following sequence alignment, the mutation was identified as a missense mutation at nucleotide 1582 in exon 11 of CACNA1S, resulting in a substitution of glycine for arginine at site 528 in CACNA1S (Fig. 2B and C). Two additional mutations observed in the same fragment were confirmed as same-sense mutations (Fig. 2B). In pedigree D (Fig. 3A), a substitution mutation of thymine to cytosine was identified in the 250 base pair SCN4A fragment containing amino acid sites 669 and 672 . Following sequence alignment, the mutation was confirmed as a missense mutation at nucleotide 2012 in exon 12 of SCN4A, resulting in a substitution of serine for phenylalanine at site 671 (Fig. 3B and C). Two additional mutations observed in the CACNA1S fragment containing nucleotide 1239 were confirmed as same-sense mutations. No mutations in these fragments containing the common mutation sites were identified in the probands of the other three pedigrees.
Genefragments encompassing the sites of alternative reported mutations in CACNA1S and SCN4A were then sequenced in the remaining three pedigrees. In pedigree A, among 43 family members in four generations, mutations in 33 subjects, including two patients (IV8 and II3), were detected. In pedigree B, among 26 family members in three generations, mutations in 20 subjects, including four patients (II1, II3, II5 and II7), were detected. In pedigree $\mathrm{E}$, among 18 family members in four generations, mutations in 12 subjects, including one confirmed patient (III7) and one suspected patient (III5), were detected. No mutations previously identified in exons 11, 20, 21, 22, 26 and 30 of CACNA1S, and in exons 5, 12, 18 and 19 of SCN4A were identified in these three pedigrees. Sequencing maps, ranging from nucleotide 1567-1596 in exon 11 of CACNA1S, and from nucleotide 1999-2028 in exon 12 of SCN4A, are presented in Figs.4 and 5, respectively.

\section{Discussion}

HPP is a disease detrimental to the quality of life of the patients and can lead to death resulting from unexpected respiratory paralysis or arrhythmia $(9,10)$. Due to variation in the age of 


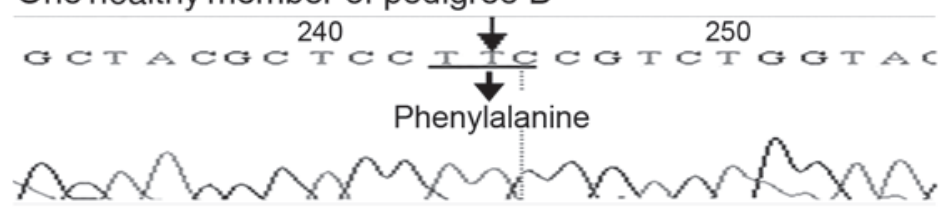

Proband of pedigree $D$

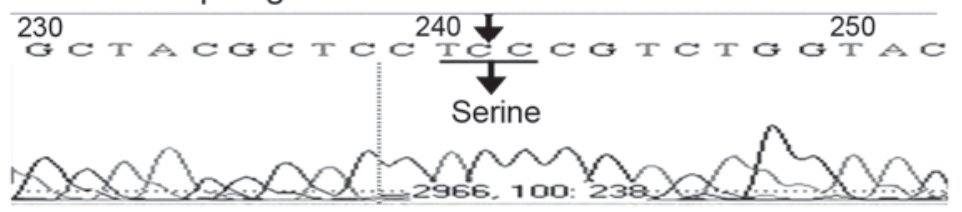

B

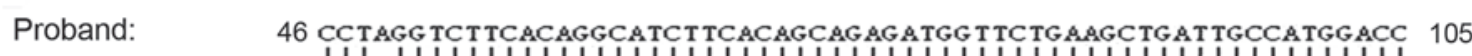

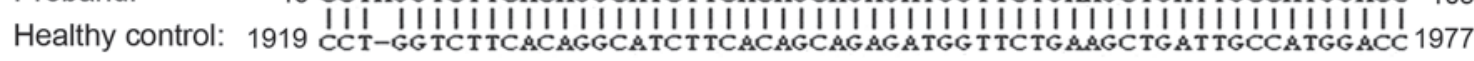

Proband: $\quad 106$ CCTACGAG TAT TTCCAGCAGGG T TGGAATATCT TCGACAGCATCATCG TCACCCTCAGCC 165 Healthy control: 1978 CCTACGAGTATTCCAGCAGGG TIMGATATCTTCGACAGCATCATCG TCACCCTCAGCC 2037

Proband: 166 TGG TAGAGCTAGGCCTGGCCAACG TACAGGGACTG TCTG TGCTACGCTCCTCCCG TCTG 224

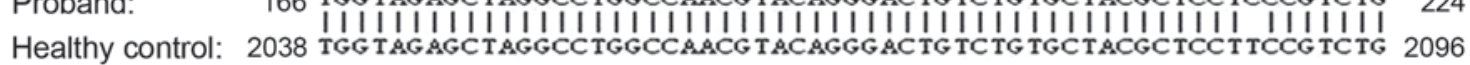

C

Proband:

VF TG IF T AEMVLL I AMDPYEYFQQGWNIFDSI IVTLSL VEL GL ANVQGL SVLRSSRLV VF TG IF TAEMYLKL I AMDPYEYFQQGWNIFDSIIVTLSL VELGLANVQGL SWLRS RL+ Healthy control: 616 VF TG IF TAEMVKLIAMPYEYFQQGWNIFDSIIVTLSL VELGLANVQGL SWLRSFRLL 674

Figure 3. Sequence analysis of SCN4A region encoding amino acid sites 669 and 672 in pedigree D. (A) Sequencing maps of the amplified fragment in the proband and one healthy member of pedigree D. (B) Sequence alignment. The sequence of the healthy control from GenBank. (C) The change in the amino acid sequence resulting from the missense mutation of thymine to cytosine at nucleotide 2012 in SCN4A. SCN4A, voltage-gated sodium channel type IV alpha subunit.
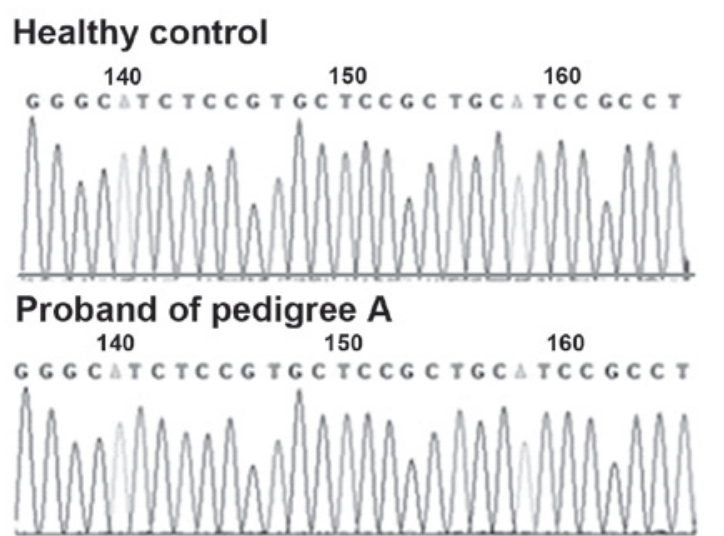

Proband of pedigree $B$

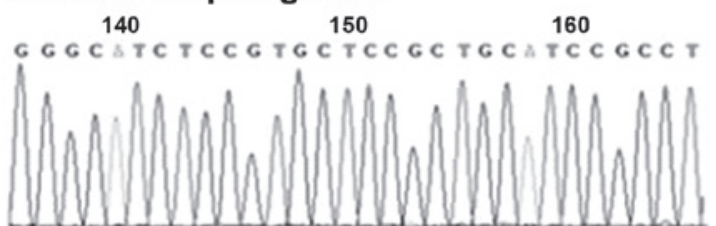

Proband of pedigree $\mathrm{E}$

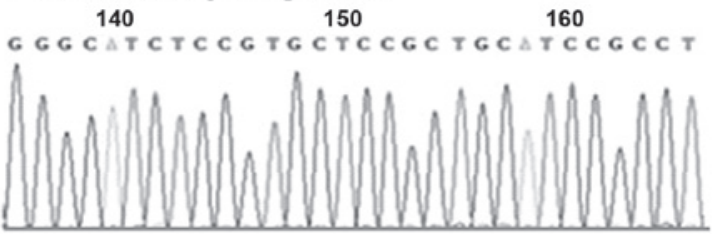

II3 of pedigree $A$

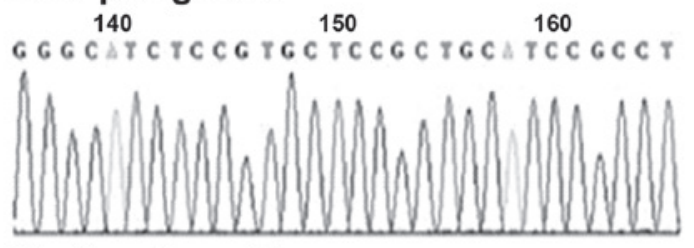

Il1 of pedigree $B$

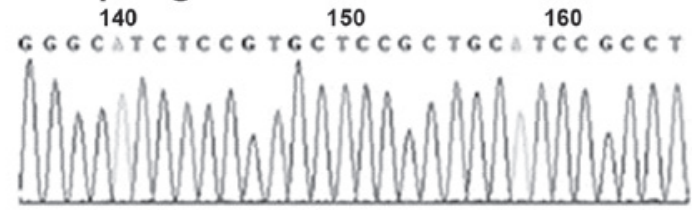

II5 of pedigree B

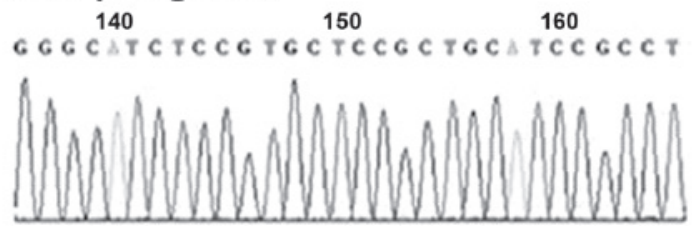

\section{II7 of pedigree $\mathrm{B}$}

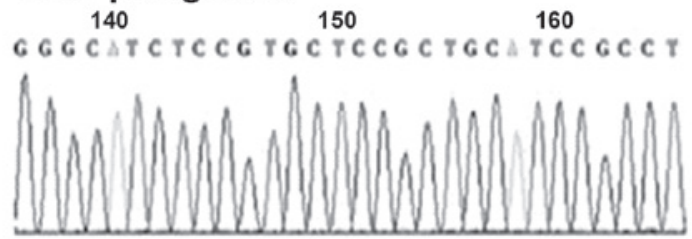

Figure 4. Sequence maps of bases from sites 1567-1596 in exon 11 of CACNA1S in a healthy control, probands and other patients of pedigrees A, B and E. CACNA1S, voltage-dependent L type calcium channel subunit alpha $1 \mathrm{~S}$. 
Table III. Verified mutation sites in CACNA1S and SCN4A in patients with hypokalemic periodic paralysis.

\begin{tabular}{lll}
\hline Gene & Exon number & \multicolumn{1}{c}{ Mutation sites } \\
\hline CACNA1S & 11 & R528H (6), R528G (6) \\
& 20 & V876E (11), R897S (12) \\
& 21 & R900G (13), R900S (6) \\
& 22 & H916Q (14) \\
SCN4A & 26 & R1086C (6) \\
& 30 & R1239H (6), R1239G (6) \\
& 5 & R222W (6), R669H (6) \\
& 12 & F671S (6), R672H (6), R672G (6), \\
& 18 & R672C (6), R672S (6) \\
& 19 & R1132Q (6), R1135H (6) \\
\hline
\end{tabular}

CACNA1S, voltage-dependent L type calcium channel subunit alpha 1S; SCN4A, voltage-gated sodium channel type IV alpha subunit; R, arginine; G, glycine; H, histidine; S, serine; C, cysteine; Q, glutamine; P, proline; V, valine; E, glutamic acid; W, tryptophan; F, phenylalanine.

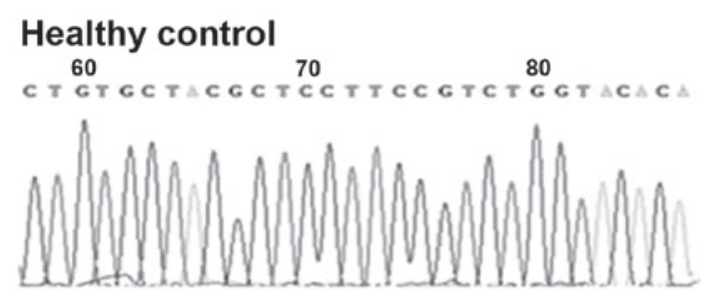

Proband of pedigree $\mathbf{A}$

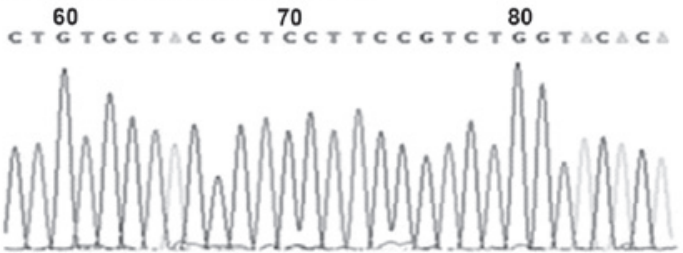

Proband of pedigree $B$
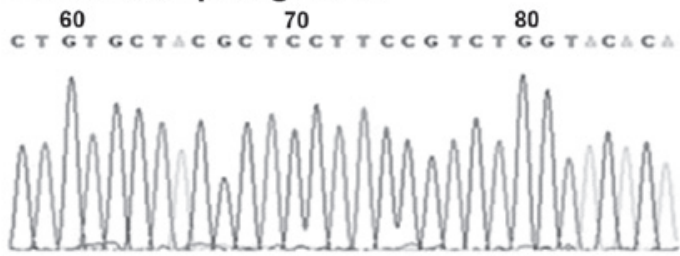

Proband of pedigree $\mathrm{E}$

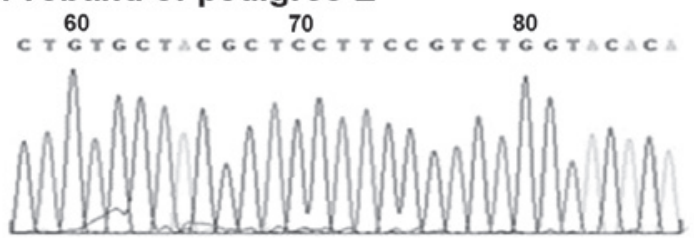

II3 of pedigree $A$

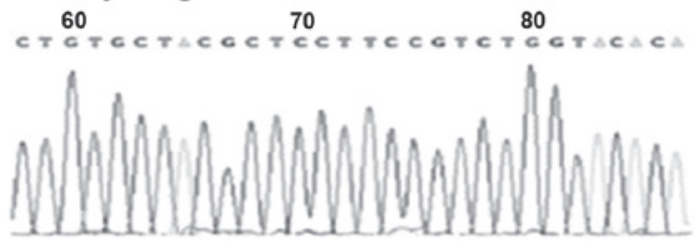

II1 of pedigree $B$

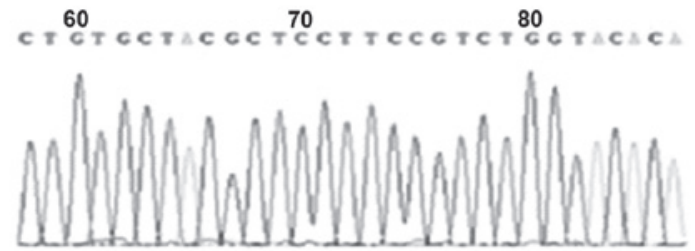

II5 of pedigree $\mathrm{B}$

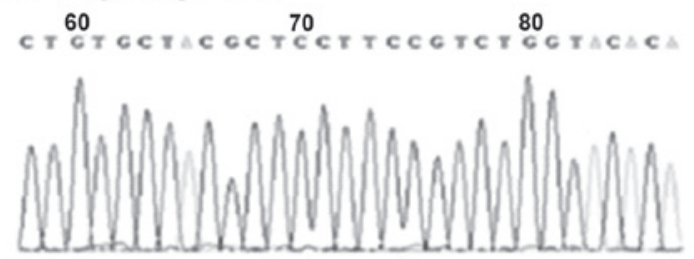

II7 of pedigree $B$

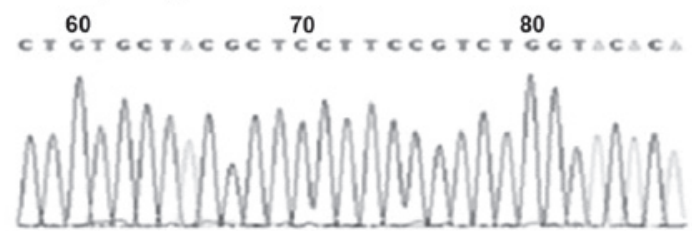

Figure 5. Sequence maps of bases from sites 1999-2028 in exon 12 of SCN4A in a healthy control, probands and other patients of pedigrees A, B and E. SCN4A, voltage-gated sodium channel type IV alpha subunit.

onset, duration, frequency and severity of attacks, the diagnosis of HPP is predominantly made according to clinical history, attack characteristics and serum potassium levels during attacks (16). Although the specific mechanism of HPP remains unclear, mutations in genes encoding ion channels in skeletal muscle have been suggested to be involved (17), and genetic analysis may aid in elucidating the pathogenesis of the disease. The inheritance pattern of HPP is known to be autosomal dominant, however it has incomplete penetrance, particularly in females (16). With several family members in the same pedigree suffering HPP, the incomplete penetrance of pathogenic genes is considered to be involved in the atavism of the pedigrees in the current study. 
Previous studies have indicated the familial transmission of HPP-associated mutations $(2,18)$. Although SCN4A mutations are also involved $(19,20)$, mutations in CACNA1S occur primarily in patients with HPP. In a study involving 58 HPP pedigrees (2), the rate of mutation detection was $77.6 \%$, including 40 pedigrees with CACNA1S mutations $(69.0 \%)$ and five pedigrees with SCN4A mutations $(8.6 \%)$ (no mutations were observed in the remaining 13 pedigrees). The two most common mutations in CACNA1S were R528H and $\mathrm{R} 1239 \mathrm{H}$, which account for 45.0 and $24.0 \%$ of mutations detected, respectively. In an additional study involving 83 cases of HPP (6), the rate of mutation was $87.9 \%$, including 65 cases with CACNA1S mutations, accounting for $78.3 \%$, and eight cases with SCN4A mutations, accounting for $9.6 \%$.

Compared with Western populations, mutations observed in Eastern populations are very similar, however the rate of mutation detection is markedly lower. In a study of Chinese patients (21), one case with the $\mathrm{R} 1239 \mathrm{H}$ mutation and two cases with the $\mathrm{R} 672 \mathrm{H}$ mutation were identified in the probands of 14 pedigrees, the mutation rate of which was $21.4 \%$, and only one case with the R672C mutation was observed in 71 sporadic patients. Mutation screening in Taiwanese patients with HPP detected one case with $\mathrm{R} 528 \mathrm{H}$ in 12 sporadic patients, accounting for $8.3 \%$, with no cases detected in 36 patients with THPP (7). An additional study in Taiwanese patients reported four cases with mutations among 60 sporadic patients with HPP, including one case with R1239H, two cases with $\mathrm{R} 669 \mathrm{H}$ and one case with $\mathrm{R} 1135 \mathrm{H}$, with the mutation rate in the current study at $6.6 \%$ (22). Mutations have been rarely reported in populations from China $(23,24)$. Accordingly, the present study additionally demonstrated a lower rate of HPP-associated gene mutations.

The known mutations in CACNA1S and SCN4A are presented in Table III. The majority of mutation sites in the table have been identified in Western populations, excluding R528G, R900G, R916Q and R1239G. In studies of Asian populations, mutations in CACNA1S were predominantly reported in case studies $(9,13,25-31)$ and pedigree studies (7,14,32-34). In the current study, a rare R528G mutation in CACNA1S in a Chinese HPP pedigree was observed, and this mutation has been previously reported in another Chinese pedigree with HPP (27). THPP shares certain common characteristics with HPP, however, the identification of pathogenic genes between them are not similar (35-37). In contrast with HPP, mutations in CACNA1S are not associated with THPP (35). To date, gene mutations have been confirmed in three THPP pedigrees, including a pedigree with an R83H mutation in KCNE3 (38), a pedigree with an M58V mutation in KCNE4 (36), and a Chinese pedigree with an F671S mutation in SCN4A (the current study). R83H and M58V have not been identified in Chinese patients with THPP, however it remains controversial whether $\mathrm{R} 83 \mathrm{H}$ is a causative mutation in THPP, due to the fact that it is additionally present in $2 \%$ of healthy individuals (39).

In conclusion, mutations in CACNA1S and SCN4A are relatively rare in Chinese HPP cases compared with cases in Western individuals. This indicates a racial difference and the possibility of other pathogenic genes or factors in Chinese patients. Due to the fact that the sample sizes in Asian pedigree studies have been relatively small, and the newly identified mutations in Chinese cases are often identified in case studies $(13,14)$, further research is required to investigate whether R528G in HPP and F671S in THPP are common in Chinese populations. Future studies should aim to identify novel mutations in Chinese patients in CACNA1S and SCN4A in addition in other genes, and to establish animal models with known mutations to further clarify the pathophysiological mechanisms of HPP.

\section{Acknowledgements}

The current study was supported by the National Natural Science Foundation of China (grant nos. 30671006 and 81170800). The authors would like to thank Edanz China for the English Language Service.

\section{References}

1. Kawamura S, Ikeda Y, Tomita K, Watanabe N and Seki K: A family of hypokalemic periodic paralysis with CACNA1S gene mutation showing incomplete penetrance in women. Intern Med 43: 218-222, 2004.

2. Sternberg D, Maisonobe T, Jurkat-Rott K, Nicole S, Launay E, Chauveau D, Tabti N, Lehmann-Horn F, Hainque B and Fontaine B: Hypokalaemic periodic paralysis type 2 caused by mutations at codon 672 in the muscle sodium channel gene SCN4A. Brain 124: 1091-1099, 2001.

3. Links TP, Ginjaar HB and van der Hoeven JH: From gene to diseases; hypokalemic periodic paralysis. Ned Tijdschr Geneeskd 148: 1035-1038, 2004 (In Dutch).

4. Kuzmenkin A, Muncan V, Jurkat-Rott K, Hang C, Lerche H, Lehmann-Horn F and Mitrovic N: Enhanced inactivation and $\mathrm{pH}$ sensitivity of $\mathrm{Na}(+)$ channel mutations causing hypokalaemic periodic paralysis type II. Brain 125: 835-843, 2002.

5. Ng WY, Lui KF, Thai AC and Cheah JS: Absence of ion channels CACN1AS and SCN4A mutations in thyrotoxic hypokalemic periodic paralysis. Thyroid 14: 187-190, 2004.

6. Matthews E, Labrum R, Sweeney MG, Sud R, Haworth A, Chinnery PF, Meola G, Schorge S, Kullmann DM, Davis MB, et al: Voltage sensor charge loss accounts for most cases of hypokalemic periodic paralysis. Neurology 72: 1544-1547, 2009.

7. Lin SH, Hsu YD, Cheng NL and Kao MC: Skeletal muscle dihydropyridine-sensitive calcium channel (CACNA1S) gene mutations in chinese patients with hypokalemic periodic paralysis. Am J Med Sci 329: 66-70, 2005.

8. Lane AH, Markarian K and Braziunene I: Thyrotoxic periodic paralysis associated with a mutation in the sodium channel gene SCN4A. J Pediatr Endocrinol Metab 17: 1679-1682, 2004.

9. Kil TH and Kim JB: Severe respiratory phenotype caused by a de novo Arg528Gly mutation in the CACNA1S gene in a patient with hypokalemic periodic paralysis. Eur J Paediatr Neurol 14: 278-281, 2010.

10. Stunnenberg BC, Deinum J, Links TP, Wilde AA, Franssen H and Drost G. Cardiac arrhythmias in hypokalemic periodic paralysis: Hypokalemia as only cause? Muscle Nerve 50: 327-332, 2014.

11. Ke T, Gomez CR, Mateus HE, et al. Novel CACNA1S mutation causes autosomal dominant hypokalemic periodic paralysis in a South American family. J Hum Genet 54: 660-664, 2009.

12. Chabrier S, Monnier N, Lunardi J. Early onset of hypokalaemic periodic paralysis caused by a novel mutation of the CACNA1S gene. J Med Genet 45: 686-688, 2008.

13. Hirano M, Kokunai Y, Nagai A, Nakamura Y, Saigoh K, Kusunoki S and Takahashi MP: A novel mutation in the calcium channel gene in a family with hypokalemic periodic paralysis. J Neurol Sci 309: 9-11, 2011.

14. Li FF, Li QQ, Tan ZX, Zhang SY, Liu J, Zhao EY, Yu GC Zhou J, Zhang LM and Liu SL: A novel mutation in CACNA1S gene associated with hypokalemic periodic paralysis which has a gender difference in the penetrance. J Mol Neurosci 46: 378-383, 2012.

15. Webb J, Cannon SC. Cold-induced defects of sodium channel gating in atypical periodic paralysis plus myotonia. Neurology 70 : 755-761, 2008. 
16. Vicart S, Sternberg D, Arzel-Hézode M, Franques J, Bendahhou S, Lory P, Hainque B, Fournier E, Nicole S and Fontaine B. Hypokalemic Periodic Paralysis. In GeneReviews ${ }^{\circledR}$ [Internet]. Pagon RA, Adam MP, Ardinger HH, Wallace SE, Amemiya A, Bean LJH, Bird TD, Dolan CR, Fong CT, Smith RJH and Stephens K (eds). University of Washington, Seattle, WI, 2002 (updated 2014).

17. Matthews E and Hanna MG: Muscle channelopathies: Does the predicted channel gating pore offer new treatment insights for hypokalaemic periodic paralysis? J Physiol 588: 1879-1886, 2010.

18. Surtees R. Inherited ion channel disorders. Eur J Pediatr 159: S199-S203, 2000.

19. Incecik F, Hergüner MO, Altunbaşak $S$ and Lehman-Horn F: Hypokalemic periodic paralysis due to the SCN4A R672H mutation in a Turkish family. Turk J Pediatr 52: 409-410, 2010.

20. Park YH and Kim JB: An atypical phenotype of hypokalemic periodic paralysis caused by a mutation in the sodium channel gene SCN4A. Korean J Pediatr 53: 909-912, 2010.

21. Ke QWW, Xu QG, Huang DH, Yu SY and Huang XS: Correlating phenotype and genotype in the familial hypokalemic periodic paralysis. Chin J Neurol 39: 323-327, 2006.

22. Sung CC, Cheng CJ, Lo YF, Lin MS, Yang SS, Hsu YC and Lin SH: Genotype and phenotype analysis of patients with sporadic periodic paralysis. Am J Med Sci 343: 281-285, 2012.

23. Kung AW, Lau KS, Fong GC and Chan V: Association of novel single nucleotide polymorphisms in the calcium channel alpha 1 subunit gene $(\mathrm{Ca}(\mathrm{v}) 1.1)$ and thyrotoxic periodic paralysis. J Clin Endocrinol Metab 89: 1340-1345, 2004.

24. Kung AW, Lau KS, Cheung WM and Chan V: Thyrotoxic periodic paralysis and polymorphisms of sodium-potassium ATPase genes. Clin Endocrinol (Oxf) 64: 158-161, 2006.

25. Kusumi M, Kumada H, Adachi Y and Nakashima K: Muscle weakness in a Japanese family of Arg1239His mutation hypokalemic periodic paralysis. Psychiatry Clin Neurosci 55: 539-541, 2001.

26. Kim JB, Lee KY and Hur JK: A Korean family of hypokalemic periodic paralysis with mutation in a voltage-gated calcium channel (R1239G). J Korean Med Sci 20: 162-165, 2005.

27. Wang Q, Liu M, Xu C, Tang Z, Liao Y, Du R, Li W, Wu X Wang X, Liu P, et al: Novel CACNA1S mutation causes autosomal dominant hypokalemic periodic paralysis in a Chinese family. J Mol Med Berl 83: 203-208, 2005.

28. Ke Q, Wu WP, Guo XH, Xu QG, Huang DH, Mao YL and Huo CN: R1239H mutation of CACNA1S gene in a Chinese family with hypokalaemic periodic paralysis. Zhonghua Yi Xue Yi Chuan Xue Za Zhi 23: 272-274, 2006 (In Chinese).
29. Kageyama K, Terui K, Tsutaya S, Matsuda E, Shoji M, Sakihara S, Nigawara T, Takayasu S, Moriyama T, Yasujima M, et al: Gene analysis of the calcium channel 1 subunit and clinical studies for two patients with hypokalemic periodic paralysis. J Endocrinol Invest 29: 928-933, 2006.

30. Ke Q, Xu QG, Huang DH, Yuan HJ, Zhao YL and Wu WP: The mutation R672H in SCN4A gene exists in Chinese patients with hypokalaemic periodic paralysis. Zhonghua Yi Xue Za Zhi 86: 724-727, 2006 (In Chinese).

31. Kim H, Hwang H, Cheong HI and Park HW: Hypokalemic periodic paralysis; two different genes responsible for similar clinical manifestations. Korean J Pediatr 54: 473-476, 2011.

32. Kim JB, Kim MH, Lee SJ, Kim DJ and Lee BC: The genotype and clinical phenotype of Korean patients with familial hypokalemic periodic paralysis. J Korean Med Sci 22: 946-951, 2007.

33. Wang W, Jiang L, Ye L, Zhu N, Su T, Guan L, Li X and Ning G: Mutation screening in Chinese hypokalemic periodic paralysis patients. Mol Genet Metab 87: 359-363, 2006.

34. Kim SH, Kim UK, Chae JJ, Kim DJ, Oh HY, Kim BJ and Lee CC Identification of mutations including de novo mutations in Korean patients with hypokalaemic periodic paralysis. Nephrol Dial Transplant 16: 939-944, 2001.

35. Dias da Silva MR, Cerutti JM, Tengan CH, Furuzawa GK, Vieira TC, Gabbai AA and Maciel RM: Mutations linked to familial hypokalaemic periodic paralysis in the calcium channel alphal subunit gene (Cav1.1) are not associated with thyrotoxic hypokalaemic periodic paralysis. Clin Endocrinol (Oxf) 56: 367-375, 2002.

36. Silva MR, Chiamolera MI, Kasamatsu TS, Cerutti JM and Maciel RM: Thyrotoxic hypokalemic periodic paralysis, an endocrine emergency: Clinical and genetic features in 25 patients. Arq Bras Endocrinol Metabol 48: 196-215, 2004 (In Portuguese).

37. Lin SH and Huang CL: Mechanism of thyrotoxic periodic paralysis. J Am Soc Nephrol 23: 985-988, 2012.

38. Dias Da Silva MR, Cerutti JM, Arnaldi LA and Maciel RM: A mutation in the KCNE3 potassium channel gene is associated with susceptibility to thyrotoxic hypokalemic periodic paralysis. J Clin Endocrinol Metab 87: 4881-4884, 2002.

39. Sternberg D, Tabti N, Fournier E, Hainque B and Fontaine B: Lack of association of the potassium channel-associated peptide MiRP2-R83H variant with periodic paralysis. Neurology 61: $857-859,2003$ 